\title{
DESIGNING MANUFACTURABLE VISCOELASTIC DEVICES USING A TOPOLOGY OPTIMIZATION APPROACH WITHIN A TRULY-MIXED FEM FRAMEWORK
}

\author{
Paolo Venini $^{1}$, Marco Pingaro ${ }^{2}$, and Carlo Cinquini ${ }^{1}$ \\ ${ }^{1}$ University of Pavia, Department of Civil Engineering and Architecture \\ via Ferrata, 3 - 27100 Pavia, ITALY \\ e-mail: \{paolo.venini,carlo.cinquini\}@ unipv.it \\ ${ }^{2}$ IUSS - Istituto Universitario di Studi Superiori di Pavia \\ Palazzo del Broletto - Piazza della Vittoria n.15, 27100 Pavia, ITALY \\ e-mail: marco.pingaro@iusspavia.it
}

Keywords: $H_{\infty}$-norm topology optimization, Viscoelasticity, Truly-mixed FEM, Additive manufacturing.

\begin{abstract}
A new approach to topology optimization is presented that is based on the minimization of the input/output transfer function $H_{\infty}$ norm. Additionally, by properly selecting input and output vector, the approach is recognized to minimize an entirely new definition of frequency-based dynamic compliance. The method is applied to viscoelastic systems in plane strain conditions that are investigated by using the Arnold-Winther finite-element resorting to a generalized solid phenomenological model. Preliminary indications on how to address the actual manufacturability of the optimal specimen are eventually outlined.
\end{abstract}




\section{INTRODUCTION}

Research on topology optimization of dynamic response dates back to the nineties and was at that time mostly intended as eigenvalue optimization [1] within a general max-min strategy that aims at maximizing the lowest eigenvalues and the like. The maximization of single and multiple eigenfrequencies and relevant band-gaps for vibrating structures is addressed in [2] whereas a similar problem for Euler-Bernoulli beams has been proposed in [3]. More recently methods for the optimal design of forced beams and continua in dynamic regime have been presented that may be roughly grouped into two main categories: approaches that make use of the dynamic compliance concept, see [4],[5] and [6] among others, and frequency domain methods that are investigated in [7], [8] and [9] just to mention a few.

Focus of this paper is on the proposal of a new frequency-based topology optimization strategy for dynamic structures that aims at the minimization of the input/output transfer function $\mathrm{H}_{\infty^{-}}$ norm. The distinctive feature of the proposed approach is that it seems to represent the natural extension to dynamics of by now classical methods for static topology optimization, [10]. Furthermore, by a proper selection of system input and output vectors, a new concept of frequencydomain compliance for dynamic systems is gathered and ready for topology optimization. Crucial for the success of the proposed methodology is the development of a semi-analytic formula for computing the gradient of the $H_{\infty}$ norm with respect to the design parameter vector (element densities) that represents an extension of the formula presented in [11]. The proposed topology optimization strategy is quite general and may be actually applied to any kind of dynamical system in state-space (or equivalently transfer-function) form such as standard elastic systems whose state-space vector encompasses displacements and velocities. However, for this paper sake a new class of linear 2D viscoelastic systems is considered along the path suggested in [12] that uses a weak-symmetry stress finite-element as opposed to what is done herein where the truly-element proposed in [13] is adopted. By using velocities (instead of displacements) as kinematic variables, the state vector of the resulting dynamical system includes velocities themselves and stresses and this makes the numerical analysis peculiar with respect to more classical elastic idealizations. The same class of viscoelastic systems was investigated in [14] wherein the the focus of the design was on stress-constraints and incompressibility.

Eventually, a few considerations on the extensions needed by the formulation to comply with additive manufacturing requirements are highlighted, mainly following the general framework proposed by Sigmund and coworkers in [15] and [16]. As to optimal design of viscoelastic materials exhibiting extreme properties, reference is made to [17]. Numerical investigations on minimum compliance problems are presented and discussed to validate the general framework.

\section{A NEW $H_{\infty}$-NORM FRAMEWORK FOR TOPOLOGY OPTIMIZATION}

\section{1 (Descriptor) state space (D)SS and transfer function TF repsentations}

The class of dynamical systems to be investigated herein may be given the time domain descriptor state-space format

$$
\begin{cases}\boldsymbol{E} \dot{\boldsymbol{x}} & =\boldsymbol{A x}+\boldsymbol{B} \boldsymbol{w} \\ \boldsymbol{z} & =\boldsymbol{C x}\end{cases}
$$

or Laplace domain, transfer-function representation

$$
\boldsymbol{Z}=\boldsymbol{G}(s) \boldsymbol{W}, \text { where: } \boldsymbol{G}(s)=\boldsymbol{C}(s \boldsymbol{E}-\boldsymbol{A})^{-1} \boldsymbol{B},
$$


where $\boldsymbol{x}, \boldsymbol{z}$ and $\boldsymbol{w}$ are the state, output and load vectors respectively, $\boldsymbol{E}$ is (an extended version of) the mass matrix, $\boldsymbol{A}$ is the structural state matrix, $\boldsymbol{B}$ a topological matrix distributing the loads to the degrees-of-freedom, $C$ the output matrix that basically selects the components of the state vector whose response need be optimized, and $Z$ and $\boldsymbol{W}$ the Laplace transforms of $\boldsymbol{z}$ and $\boldsymbol{w}$, respectively. It is assumed that matrices $\boldsymbol{E}$ and $\boldsymbol{A}$ depend on a design-variable vector $\boldsymbol{p}$, i.e. in explicit form one writes

$$
\boldsymbol{E}=\boldsymbol{E}(\boldsymbol{p}), \boldsymbol{A}=\boldsymbol{A}(\boldsymbol{p}) .
$$

\section{2 $\quad H_{\infty}$ norm definition and physical meaning}

A systematic exposition of the $H_{\infty}$ norm concept is beyond the scopes of this paper and reference is made to [18] for a in-depth treatment including applications to robust control of uncertain dynamical systems. The transfer-function representation of Equation 2 is referred to but all the reasoning applies to the state-space form of Equation 1 as well. To start with we consider the simpler case of a single-input/single-output system (that may be however be characterized by a multidimensional state vector $\boldsymbol{x}$ ). The $H_{\infty}$ norm of a single-input/singleoutput (SISO) linear system with given transfer function $\boldsymbol{G}(s)$ is defined as the peak gain of the frequency response, i.e.

$$
\|\boldsymbol{G}\|_{\infty}=\sup _{\omega}|\boldsymbol{G}(i \omega)| .
$$

Recalling that $|\boldsymbol{G}(i \omega)|$ is the factor by which the amplitude of a sinusoidal input with angular frequency $\omega$ is magnified by the system, it is seen that the $H_{\infty}$ norm is a measure of the largest factor by which any sinusoid is magnified by the system. In this respect the $H_{\infty}$ norm of a dynamical system represents the natural extension to dynamics of static response measures in that it gives the analyst the maximum system response all over the frequency range in the spirit of Fourier analysis. Switching now to multi-input/multi-output (MIMO) systems, the $H_{\infty}$ norm is the peak gain across all input/output channels. For an $n \times m$ transfer function matrix $\boldsymbol{G}(s)$, a natural way to achieve this is to introduce the maximum gain of $\boldsymbol{G}(i \omega)$ at the frequency $\omega$. Let $\boldsymbol{b}=\left[v_{1}, \ldots, v_{m}\right]^{T} \in C^{m}$ be a complex-valued vector with Euclidean norm

$$
\|\boldsymbol{b}\|_{2}=\left(\left|v_{1}\right|^{2}+\cdots+\left|v_{m}\right|^{2}\right)^{1 / 2} .
$$

The maximum gain of $G$ at frequency $\omega$ may be written as

$$
\begin{aligned}
\|\boldsymbol{G}(i \omega)\| & =\max _{\boldsymbol{b}}\left\{\frac{\|\boldsymbol{G}(i \omega) \boldsymbol{b}\|_{2}}{\|\boldsymbol{b}\|_{2}}: \boldsymbol{b} \neq \mathbf{0}, \boldsymbol{b} \in C^{m}\right\} \\
& =\max _{\boldsymbol{b}}\left\{\|\boldsymbol{G}(i \omega) \boldsymbol{b}\|_{2}:\|\boldsymbol{b}\|_{2}=1, \boldsymbol{b} \in C^{m}\right\} .
\end{aligned}
$$

Finally, the $H_{\infty}$ norm of the transfer function matrix $\boldsymbol{G}(s)$ is defined as

$$
\|\boldsymbol{G}\|_{\infty}=\sup _{\omega}\|\boldsymbol{G}(i \omega)\|
$$

One may further show that the matrix norm $\|\boldsymbol{G}(i \omega)\|$ is equal to the maximum singular value $\bar{\sigma}(\boldsymbol{G}(i \omega))$ of the matrix $\boldsymbol{G}(i \omega)$. Therefore, the $H_{\infty}$ norm may also be written as

$$
\|\boldsymbol{G}\|_{\infty}=\sup _{\omega} \bar{\sigma}(\boldsymbol{G}(i \omega)) .
$$


A few approaches for computing the $H_{\infty}$-norm of a dynamical system are available in the literature, see e.g. [18], whose analysis is beyond the scopes of this paper. It suffices here to say that a few methods have been tested within the MATLAB [19] based code developed for the numerical simulations to be presented next that displayed comparable performance in terms of accuracy and speed. The MATLAB functions getPeakGain and hinfnorm have been actually used that, for a given dynamical system saved in descriptor state-space (dss) or transfer-function ( $\mathrm{t} f$ ) formats, return its $H_{\infty}$ norm and the frequency $\omega$ at which the peak gain takes place that are needed for the computation of the relevant gradient vector $\frac{\partial\|\boldsymbol{G}\|_{\infty}}{\partial \boldsymbol{p}}$.

\subsection{The abstract topology optimization problem}

The abstract topology optimization problem dealt with hereinafter may therefore be written as

$$
\begin{cases}\min _{\boldsymbol{p}} F(\boldsymbol{p})= & \|\boldsymbol{G}(i \omega, \boldsymbol{p})\|_{\infty} \\ \text { s.t. } & \boldsymbol{G}(i \omega, \boldsymbol{p})=\boldsymbol{C}(i \omega \boldsymbol{E}(\boldsymbol{p})-\boldsymbol{A}(\boldsymbol{p}))^{-1} \boldsymbol{B} \\ & V(\boldsymbol{p}) \leq V_{\max } \\ & 0 \leq \boldsymbol{p} \leq 1\end{cases}
$$

where $9_{3}$ is a global volume constraint and $9_{4}$ is the usual limitation on the element densities $\boldsymbol{p}$. As to practical topology optimization problems that fit the above framework the following are worth mentioning:

1. minimization of the displacement response at selected points of the structure. With reference to Equation $1_{2}$, this may be achieved by selecting a Boolean $C$ matrix whose unitary entries are such that the product $\boldsymbol{C} \boldsymbol{x}$ extracts from the state vector $\boldsymbol{x}$ those only components of the response the designer wants to be minimized;

2. minimization of the stress response at selected points of the structure. When using a mixed finite element approximation method, the goal is achieved with the very same technique as for displacement response since stresses $\boldsymbol{\sigma}$ belong to the state vector $\boldsymbol{x}$ as well. Furthermore, when a mixed finite element approach is adopted the output vector $z$ may include both displacements and stresses so as to end up with an optimal structure with respect to kinematic and static quantities at the same time;

3. minimization of (a new concept of) dynamic compliance. Dynamic compliance has recently received much attention by the engineering community [5]. The classical static concept of compliance is typically extended to dynamics by choosing an objective functions that is the integral average over a finite time interval of the product of the loads times dual (in virtual work sense) displacements. In the spirit of the proposed approach, a new concept of dynamic compliance is introduced herein that is suitable for numerical investigations. Reference is again made to Equation $1_{2}$ that provides the system output vector in the form $\boldsymbol{z}=\boldsymbol{C} \boldsymbol{x}$. By considering a single-output system (in which case $\boldsymbol{C}$ is a single-line vector) whose entries are the load intensities $F_{i}$ positioned at the same entries as the dual displacements $u_{i}$, one gets a single-output of type $\boldsymbol{z}=\boldsymbol{C} \boldsymbol{x}=\sum_{i} F_{i} u_{i}$, i.e. the system dynamic compliance $\mathscr{C}$. By this selection of matrix $C$ one may then get a topologically optimal structure that minimizes the system compliance over the entire frequency range of the acting loads. 


\subsection{Computing the gradient $\frac{\partial\|\boldsymbol{G}(i \omega, \boldsymbol{p})\|_{\infty}}{\partial \boldsymbol{p}}$}

Computing the $H_{\infty}$-norm sensitivity of a transfer function matrix with respect to a plant parameter may be shown to be quite an hard task that, at least in principle, may be accomplished via a finite-difference approximation as long as the system does not depend on too many parameters, as is unfortunately the case for a structure undergoing topology optimization. To make the optimization procedure feasible, a fast and reliable semi-analytical approach is therefore necessary for the numerical procedure to convergence (herein we use the Method of Moving Asymptotes [20]). Not many algorithms are available in the literature and [11] seems to represent the only contribution on this delicate subject. First of all, the transfer function matrix in Equation (2) is re-written by posing $s=j \omega$, i.e.

$$
\boldsymbol{G}(i \omega, \boldsymbol{p})=\boldsymbol{C}(i \omega \boldsymbol{E}(\boldsymbol{p})-\boldsymbol{A}(\boldsymbol{p}))^{-1} \boldsymbol{B} .
$$

If now $\boldsymbol{M}(\boldsymbol{p})$ is any differentiable and invertible matrix, a basic result in matrix differential calculus allows one to write

$$
\frac{\partial \boldsymbol{M}^{-1}}{\partial p_{j}}=-\boldsymbol{M}^{-1} \frac{\partial \boldsymbol{M}}{\partial p_{j}} \boldsymbol{M}^{-1}
$$

that, once applied to $(i \omega \boldsymbol{E}(\boldsymbol{p})-\boldsymbol{A}(\boldsymbol{p}))^{-1}$ in Equation 10, gives

$$
\frac{\partial \boldsymbol{G}(i \omega, \boldsymbol{p})}{\partial p_{j}}=-\boldsymbol{C}(i \omega \boldsymbol{E}-\boldsymbol{A})^{-1}\left[i \omega \frac{\partial \boldsymbol{E}(\boldsymbol{p})}{\partial p_{j}}-\frac{\partial \boldsymbol{A}(\boldsymbol{p})}{\partial p_{j}}\right](i \omega \boldsymbol{E}-\boldsymbol{A})^{-1} \boldsymbol{B} .
$$

A singular value decomposition of $G$ need then be computed that reads

$$
\boldsymbol{G}_{k \times m}=\boldsymbol{U} \boldsymbol{\Sigma} \boldsymbol{V}^{H}
$$

where $H$ denotes the conjugate transpose, and $\boldsymbol{U}$ and $\boldsymbol{V}$ are unitary matrices, i.e. $\boldsymbol{U}^{H} \boldsymbol{U}=\boldsymbol{I}$, $\boldsymbol{V}^{H} \boldsymbol{V}=\boldsymbol{I}$. The diagonal elements of $\boldsymbol{\Sigma}$ are called singular values of $\boldsymbol{G}$ whereas column vectors $\boldsymbol{U}_{i} \in \boldsymbol{U}$ and $\boldsymbol{V}_{i} \in \boldsymbol{V}$ are respectively called left and right singular vectors of $\boldsymbol{G}$ associated to the singular value $\sigma_{i} \in \Sigma$ :

$$
\begin{aligned}
& \boldsymbol{\Sigma}=\operatorname{diag}\left(\sigma_{1}, \sigma_{2}, \ldots, \sigma_{\ell}\right), \text { singular values }, \ell=\min (k, m) \\
& \boldsymbol{U}=\text { unitary matrix of left singular vectors } \\
& \boldsymbol{V}=\text { unitary matrix of right singular vectors }
\end{aligned}
$$

The main result arrived at in [11] (actually in the case of standard state-space system for which $\boldsymbol{E} \equiv \boldsymbol{I}$ ) is a rigorous proof of the sensitivity formula that may be shown to be written as

$$
\left.\frac{\partial\|\boldsymbol{G}(i \omega ; \boldsymbol{p})\|_{\infty}}{\partial p_{i}}\right|_{\boldsymbol{p}=\boldsymbol{p}_{0}}=\operatorname{Real}\left[\boldsymbol{U}_{1}^{H} \frac{\partial \boldsymbol{G}\left(i \omega, \boldsymbol{p}_{0}\right)}{\partial p_{i}} \boldsymbol{V}_{1}\right]
$$

where $\omega$ is the frequency at which the peak value of the transfer function matrix $G$ is experienced, i.e. the one where the $H_{\infty}$ norm is actually computed. 


\subsection{Computational issues on the $H_{\infty}$ norm and its gradient}

The computation of the $H_{\infty}$ norm of a complex (MIMO) system with a massive state-vector $\boldsymbol{x}$ is quite a complicated task that is object of active research by the control community. Depending on the complexity of the system, the computation of the $H_{\infty}$ norm may require from several minutes to half an hour as order of magnitude. However, within a topology optimization session having the $H_{\infty}$ norm as objective function, one needs to compute the $H_{\infty}$ norm (and the peak frequency) of a sequence of systems, say $\boldsymbol{G}_{n}\left(i \omega, \boldsymbol{p}_{n}\right)$ and typically the difference of the (2)norm of two consecutive design parameters $\boldsymbol{p}_{n}$ and $\boldsymbol{p}_{n+1}$ is quite small and (under the obvious hypothesis of continuity with respect to the parameters) such is therefore the difference between the $H_{\infty}$ norm of the consecutive systems $\boldsymbol{G}_{n}\left(i \omega, \boldsymbol{p}_{n}\right)$ and $\boldsymbol{G}_{n+1}\left(i \omega, \boldsymbol{p}_{n+1}\right)$ as well as that of the peak frequencies $\omega_{n}$ and $\omega_{n+1}$. This suggests the following continuation method that allows a dramatic reduction of the CPU time needed to compute the $H_{\infty}$ norm:

1. For the first two iterations, i.e. $n=1,2$, compute the exact $H_{\infty}$ norms $\left\|\boldsymbol{G}_{1}\left(i \omega, \boldsymbol{p}_{1}\right)\right\|_{\infty}$, $\left\|\boldsymbol{G}_{2}\left(i \omega, \boldsymbol{p}_{2}\right)\right\|_{\infty}$ and the relevant peak frequencies $\omega_{1}$ and $\omega_{2}$, e.g. using the resident Matlab functions getPeakGain or hinfnorm [19];

2. for each subsequent iteration $n>2$, set $\Delta \omega_{n+1}=\omega_{n}-\omega_{n-1}$ and consider the frequency interval centered on $\omega_{n}$ given as $I_{n+1}=\left(\omega_{n}-\kappa \Delta \omega_{n+1}, \omega_{n}+\kappa \Delta \omega_{n+1}\right)$, where $\kappa$ governs the amplitude of $I_{n+1}(\kappa=5$ has been used in the simulations after a quick trial-and-error procedure);

3. Sample $I_{n+1}$ with a regular grid of points (50 points are used in the computations to be presented next) and compute the frequency response of the system for all such points in the frequency domain. This amounts to a forward dynamic computation that may be accomplished using the Matlab function freqresp that is far quicker than the actual computation of $\left\|\boldsymbol{G}_{n+1}\left(i \omega, \boldsymbol{p}_{n+1}\right)\right\|_{\infty}$;

4. set $\left\|\boldsymbol{G}_{n+1}\left(i \omega, \boldsymbol{p}_{n+1}\right)\right\|_{\infty}$ equal to the maximum over all the sampled points of the absolute value of the frequency responses returned by freqresp.

It may be useful to check the procedure approximately once every 50 iterations by computing exactly the $H_{\infty}$ norm and evaluate whether the approximate strategy is stable or not (in the numerical computations to be presented next, the continuation strategy proposed has proven to be always stable).

As for the computation of the $H_{\infty}$-norm gradient, reference is made to [21] where a comparison between the CPU time needed to get a finite-difference approximation versus using Equation 15 is performed showing that Equation 15 allows for a CPU time reduction of approximately three orders of magnitude.

\section{THE VISCOELASTIC BIDIMENSIONAL SYSTEM UNDER OPTIMIZATION}

\subsection{Strong form}

The viscoelastic model originally introduced in [12] is considered that is based on a stressadditive decomposition as in Figure 1. 


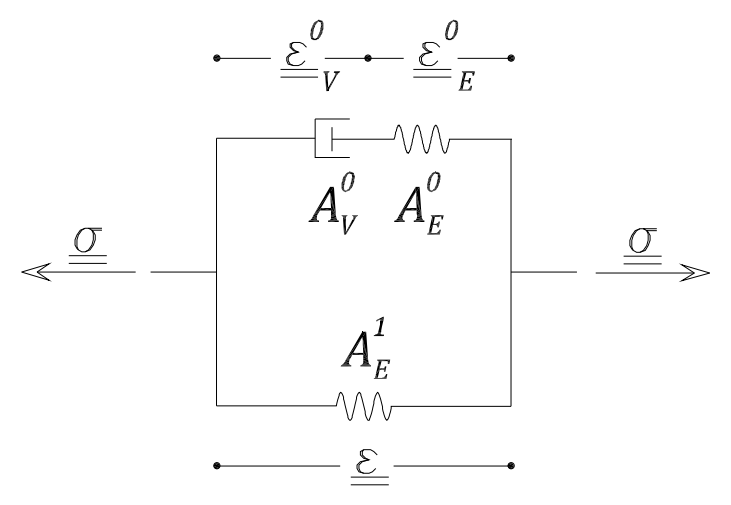

Figure 1: Standard solid phenomenological model

As usual when adopting Hellinger-Reissner variational principles, compliance tensors relating strains to stresses are introduced that allow one to write

$$
\left\{\begin{aligned}
\underline{\underline{\underline{A}}}_{E}^{0} \underline{\underline{\underline{\sigma}}}_{0}+\underline{\underline{A}}^{0} \underline{\underline{\underline{\sigma}}}_{0} & =\underline{\underline{\varepsilon}}(\underline{v}) \\
& \underline{\underline{\underline{\underline{A}}}}_{E}^{1} \underline{\underline{\underline{\sigma}}}_{1}=\underline{\underline{\varepsilon}}(\underline{v})
\end{aligned}\right.
$$

where ${\underline{\underline{\underline{A^{A}}}}}_{E}$ and $\underline{\underline{\underline{A}}}_{V}^{0}$ are the elastic and viscous compliance tensors of the viscoelastic component, $\underline{\underline{\underline{A}}}_{E}{ }^{1}$ is the elastic compliance tensor that is in parallel with the viscoelastic one and $\underline{v}$ is the velocity field. Hereinafter plane strain conditions shall be considered allowing one to write each compliance tensor in matrix form as

$$
A_{E, V}^{0,1}=\frac{1+\nu}{E_{E, V}^{0,1}(\boldsymbol{p})}\left[\begin{array}{ccc}
1-\nu & -\nu & 0 \\
-\nu & 1-\nu & 0 \\
0 & 0 & 1
\end{array}\right]
$$

where, as usual in topology optimization of isotropic structures, it is assumed that the Young modulus only depends on the material density $\boldsymbol{p}$. One should notice that a stress-velocity formulation is being used that presents several advantages over more classical stress-displacement approaches, including the ease with which dynamic effects may be considered in the analysis. Therefore, compatibility relations are written in terms of strain velocities as

$$
\underline{\underline{\varepsilon}}(\underline{v})=\underline{\underline{\nabla^{s}}} \underline{v}=\frac{1}{2}\left(\underline{\underline{\nabla v}}+\underline{\underline{\nabla v}}{ }^{T}\right)
$$

whereas the dynamic equilibrium reads

$$
-\rho \underline{\dot{v}}+\underline{\operatorname{div}} \underline{\underline{\sigma}}=-\rho \underline{g} .
$$

\subsection{Truly-mixed formulation discretized with the Arnold-Winther finite element}

By observing that the total stress $\underline{\underline{\sigma}}$ may be additively decomposed as $\underline{\underline{\sigma}}=\underline{\underline{\sigma}}_{0}+\underline{\underline{\sigma}}_{1}$, the continuous variational formulation of the problem at hand may be obtained by eliminating the strain tensor $\underline{\underline{\varepsilon}}$ in Equations 16 and 18, testing the resulting equation by two virtual stress fields 
$\underline{\underline{\tau}}, 0, \underline{\tau} 1$, and the equilibrium equation 19 by a virtual velocity field $\underline{w}$, so as to write:

Find $\left(\underline{\underline{\sigma}}_{0}, \underline{\underline{\sigma}}_{1}, \underline{v}\right) \in H(\operatorname{div}, \Omega) \times H(\operatorname{div}, \Omega) \times L^{2}(\Omega)$ such that

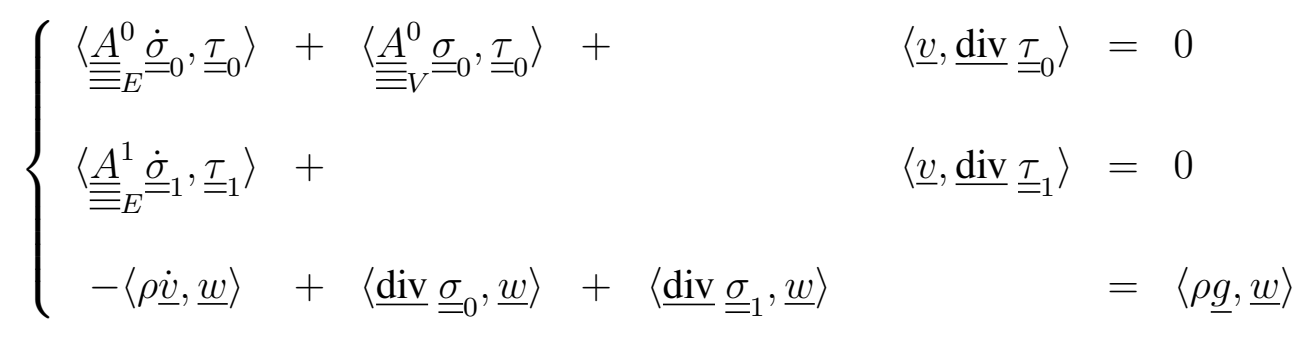

$$
\begin{aligned}
& \forall \underline{\underline{\tau}}_{0} \in H(\operatorname{div}, \Omega), \forall \underline{\underline{\tau}}_{1} \in H(\operatorname{div}, \Omega), \forall \underline{w} \in L^{2}(\Omega) .
\end{aligned}
$$

In more compact form, one may rewrite the governing system in Equation 20 in matrix-vector notation as usual within the framework of mixed methods, i.e.

$$
\left[\begin{array}{ccc}
A_{E}^{0}(\boldsymbol{p}) & 0 & 0 \\
0 & A_{E}^{1}(\boldsymbol{p}) & 0 \\
0 & 0 & -M(\boldsymbol{p})
\end{array}\right]\left[\begin{array}{c}
\boldsymbol{\sigma}_{0} \\
\boldsymbol{\sigma}_{1} \\
\boldsymbol{v}
\end{array}\right]+\left[\begin{array}{ccc}
A_{V}^{0}(\boldsymbol{p}) & 0 & B^{T} \\
0 & 0 & B^{T} \\
B & B & 0
\end{array}\right]\left[\begin{array}{c}
\boldsymbol{\sigma}_{0} \\
\boldsymbol{\sigma}_{1} \\
\boldsymbol{v}
\end{array}\right]=\left[\begin{array}{c}
0 \\
0 \\
\rho \boldsymbol{g}
\end{array}\right]
$$

The triangular Arnold-Winther finite element used in this paper is the lowest-order of the family of finite elements introduced in the pioneering paper [13]. Figure 2 shows the relevant degrees of freedom that may be listed as follows. As to the stresses, one should notice that the symmetry of the stress tensor is imposed strongly so that the components to be approximated are $\sigma_{11}, \sigma_{22}, \sigma_{12}$ and one ends up with 24 degrees of freedom:

- the 3 components of the stress tensor $\sigma_{11}, \sigma_{22}, \sigma_{12}$ at each vertex of the triangle ( 9 dofs);

- the moments of order zero and one of the traction vector $\underline{\sigma} \cdot \underline{n}$ along each edge of the triangle (12 dofs);

- the average of the the components of the stress tensor over the triangle, i.e. $\int_{T} \sigma_{11}, \int_{T} \sigma_{22}$, $\int_{T} \sigma_{12},(3$ dofs $)$.

As to the velocity vector $\underline{v}$, a standard element-wise linear globally discontinuous approximation is adopted.

\subsection{Recovering the state-space and transfer function formats}

The state-space or transfer-function format of Equations 1 or 2 may be recovered by introducing the state vector

$$
\boldsymbol{x}=\left[\begin{array}{c}
\boldsymbol{\sigma}_{0} \\
\boldsymbol{\sigma}_{1} \\
\boldsymbol{v}
\end{array}\right]
$$

and the structural matrices

$$
\boldsymbol{E}(\boldsymbol{p})=\left[\begin{array}{ccc}
A_{E}^{0}(\boldsymbol{p}) & 0 & 0 \\
0 & A_{E}^{1}(\boldsymbol{p}) & 0 \\
0 & 0 & -M(\boldsymbol{p})
\end{array}\right], \boldsymbol{A}(\boldsymbol{p})=-\left[\begin{array}{ccc}
A_{V}^{0}(\boldsymbol{p}) & 0 & B^{T} \\
0 & 0 & B^{T} \\
B & B & 0
\end{array}\right]
$$




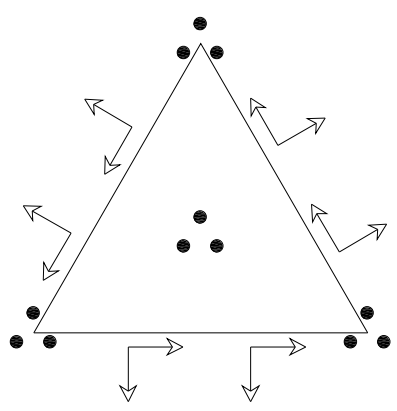

Arnold-Winther Stress DOFS

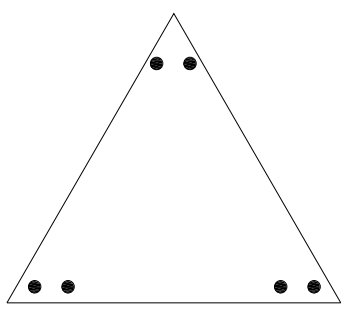

Displacements DOFS

Figure 2: Arnold-Winther DOFS

As to the loading term $\boldsymbol{B} \boldsymbol{w}$, an explicit formulation should be derived on a case by case basis but a sufficiently general possibility is to pose

$$
\boldsymbol{w}=\left[\begin{array}{c}
0 \\
0 \\
\rho \boldsymbol{g}
\end{array}\right], \quad \boldsymbol{B}=\left[\begin{array}{ccc}
\mathbf{0} & \mathbf{0} & \mathbf{0} \\
\mathbf{0} & \mathbf{0} & \mathbf{0} \\
\mathbf{0} & \mathbf{0} & \boldsymbol{I}
\end{array}\right]
$$

As to the dependence of stiffness and mass density on the design variables, as usual in topology optimization, each element is given a (mathematical) density $p \in[0,1]$ that is used within a SIMP approach [10] so as the following interpolations are considered

$$
\begin{array}{r}
E(p)=E_{\text {min }}+p^{q}\left(E_{\text {full }}-E_{\text {min }}\right) \\
\rho(p)=\rho_{\text {min }}+p\left(\rho_{\text {full }}-\rho_{\text {min }}\right),
\end{array}
$$

where the exponent $q$ is set equal to 3 as usual and Equation 25 applies to elastic and viscous phases of the material described by the standard model in Figure 1.

\section{ADDRESSING THE MANUFACTURABILITY ISSUE}

Manufacturability of the designed specimen is addressed via the three-field density approach for which reference is made to [15] for a comprehensive exposition and [16] for the physical meaning of the three involved densities within standard micro/nano lithography manufacturing processes. Many similarities of these approaches may be found with the findings of Guest and co-workers, see [22] for single-phase projection and [23] for multiple phase projection. The three density fields entering the formulation originate a chain like density transformation that reads [15]

$$
p \rightsquigarrow \widetilde{p} \rightsquigarrow \widehat{\widetilde{p}},
$$

where $p$ is the bounded mathematical density, $\widetilde{p}(p)$ is the filtered density typically obtained from $p$ by a convolution-type filter (to avoid checkerboarding among other undesirable effects) and $\widehat{\widetilde{p}}$ is the projected density that allows to remove grey regions that unavoidably affect the filtered density $\widetilde{p}$ and to impose a minimum length scale to the specimen to be designed and possibly manufactured. 


\subsection{Density and Heaviside projection filters}

A density filter is first used to switch from the mathematical density $p$ to the intermediate density $\widetilde{p}_{e}$ of the generic element $e$ that reads

$$
\widetilde{p}_{e}=\frac{1}{\sum_{i \in N_{e}} H_{e i}} \sum_{i \in N_{e}} H_{e i} p_{i}
$$

where $N_{e}$ is the set of all elements whose center-to-center distance $\Delta(e, i)$ from element $e$ is less than the prescribed filter radius $r_{\min }$ and the weighting factor $H_{e i}$ is defined as

$$
H_{e i}=\max \left(0, r_{\min }-\Delta(e, i)\right) .
$$

For this paper sake only the solid phase is then projected. To this goal, the Heaviside projection filter

$$
\widehat{\widetilde{p}}(\widetilde{p})=\left\{\begin{array}{lll}
1 & \text { if } & \widetilde{p}>0 \\
0 & \text { if } & \widetilde{p}=0
\end{array}\right.
$$

is relaxed to gain differentiability and the following filter is actually used $[22,15]$

$$
\widehat{\widetilde{p}}=1-e^{-\beta \widetilde{p}}+\widetilde{p} e^{-\beta}
$$

where $\beta=0$ means no filtering and for $\beta \rightarrow \infty$ the Heaviside filter is recovered. As suggested in $[23,24]$, a continuation method is actually implemented that starts with a low value of $\beta$ that is increased along with the iterations to impose the Heaviside filtering conditions on the final design.

\subsection{The modified topology optimization problem}

After the mathematical density $\boldsymbol{p}$ is filtered $(\widetilde{\boldsymbol{p}})$ and projected $(\widehat{\widetilde{\boldsymbol{p}}})$, the (formally) new $H_{\infty}$ topology optimization problem reads

$$
\begin{cases}\min _{\boldsymbol{p}} F(\boldsymbol{p})= & \|\boldsymbol{G}(i \omega, \boldsymbol{p})\|_{\infty} \\ \text { s.t. } & \boldsymbol{G}(i \omega, \boldsymbol{p})=\boldsymbol{C}(i \omega \boldsymbol{E}(\boldsymbol{p})-\boldsymbol{A}(\boldsymbol{p}))^{-1} \boldsymbol{B} \\ & V(\widehat{\widehat{\boldsymbol{p}}}) \leq V_{\max } \\ & 0 \leq \boldsymbol{p} \leq 1\end{cases}
$$

The sensitivity of the $H_{\infty}$ norm with respect to the design densities $\widehat{\widetilde{\boldsymbol{p}}}$ is computed using the formula in Equation 15 whereas the chain rule is used to compute gradients with respect to the initial mathematical densities $\boldsymbol{p}$, i.e.

$$
\frac{\partial F}{\partial \boldsymbol{p}}=\frac{\partial F}{\partial \widehat{\widetilde{\boldsymbol{p}}}} \frac{\partial \widehat{\widetilde{\boldsymbol{p}}}}{\partial \widetilde{\boldsymbol{p}}} \frac{\partial \widetilde{\boldsymbol{p}}}{\partial \boldsymbol{p}}
$$

where

$$
\frac{\partial \widetilde{p}_{e}}{\partial p_{j}}=\frac{1}{\sum_{i \in N_{e}} H_{e i}} H_{e j}, \quad \frac{\partial \widehat{\widetilde{p}}_{e}}{\partial \widetilde{p}_{e}}=\beta e^{-\beta \widetilde{p}_{e}}+e^{-\beta} .
$$




\subsection{Assessing the quality of the design}

The quality of the design should be assessed with respect to the following two parameters:

1. the first one is of course the value of the objective function to be minimized, i.e. the $H_{\infty}$ norm for this paper sake;

2. secondly, to check the effectiveness of the proposed strategy to cope with manufacturability issues, the following gray indicator shall be evaluated [15]

$$
M=\frac{\sum_{i=1}^{N} 4 \widehat{\widetilde{p}}(1-\widehat{\widetilde{p}})}{N},
$$

where $N$ is the dimension of vector $\widehat{\widetilde{p}}$, i.e. the number of elements, and the lower $M$ the better.

\section{Numerical study}

The bidimensional structure Figure 3 in plane strain conditions is considered with physical properties as in Table 1. A Single-Input Single-Output (SISO) realization is investigated imposing that the two loads $F_{A}$ and $F_{B}$ are acting simultaneously with the same (normalized) intensity whereas by properly choosing the topological matrix $C$ (Equation 1), the output vector $z$ (Equation 1) is given as

$$
\boldsymbol{z}=\mathscr{C}=F_{A} v_{A}+F_{B} v_{B}
$$

i.e. the the output represents the (time derivative of the) dynamic compliance exploited all over the frequency range thanks to the $H_{\infty}$-norm concept.

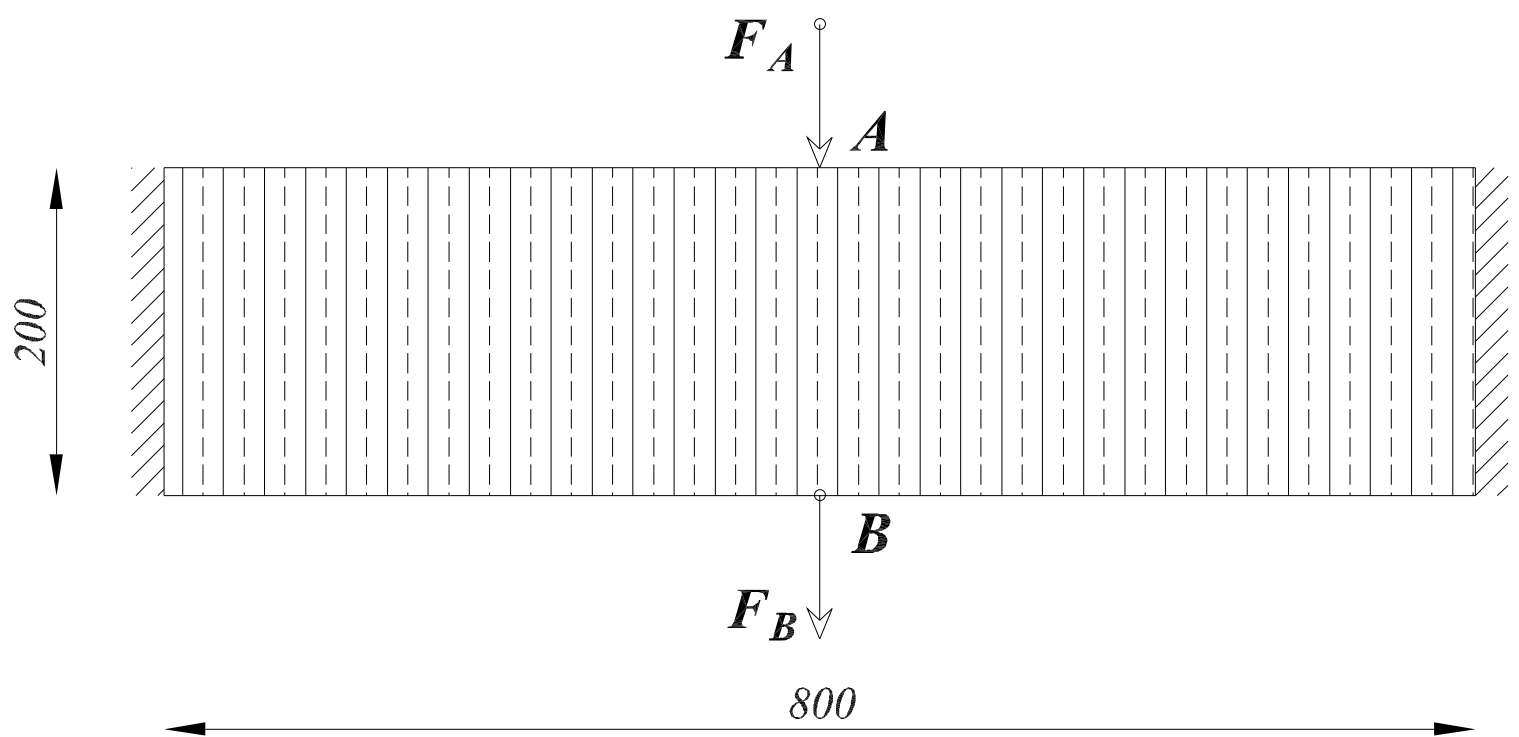

Figure 3: The bidimensional structure under optimization

Due to the symmetry of the system, only the left half is investigated by using $96 \times 48$ square cells further subdivided into 4 Arnold-Winther finite elements each. As to the overall volume 


\begin{tabular}{c|c|c|c}
\hline$E_{E}^{0}[\mathrm{Mpa}]$ & $E_{V}^{0}[\mathrm{MPa} \mathrm{s}]$ & $E_{E}^{1}[\mathrm{MPa}]$ & $\rho_{0}\left[\mathrm{~kg} / \mathrm{m}^{3}\right]$ \\
\hline 2 & 0.2 & 2 & 10 \\
\hline
\end{tabular}

Table 1: Material properties of the adopted viscoelastic material

constraint, the maximum volume $V_{\max }$ is set to $40 \%$ of the total volume. As to the continuation method to update the value of the parameter $\beta$ characterizing the projection filter (Equation 30), an initial value $\beta=2$ is considered and doubled then every 25 iterations.

Figure 4 shows the MMA performance toward the minimization of $\|\boldsymbol{G}(i \omega, \boldsymbol{p})\|_{\infty}$. Two issues are worth mentioning at this regard:

- the overall convergence path is not monotonic and remarkable oscillations take place for iterations between the 20th and the 40th;

- when the value of $\beta$ is updated the objective function decreases for that very same iteration than increases and a decreasing path shows up until $\beta$ is further updated. Such (smaller) oscillatory behavior should not be attributed to the MMA algorithm but to a volume constraint violation due to the fact that the projection filter is not volume-preserving. This violation could be prevented by a proper scaling of the filter but for this paper sake it was chosen to use the projection filter as is, and let the MMA algorithm take care of the volume constraint.

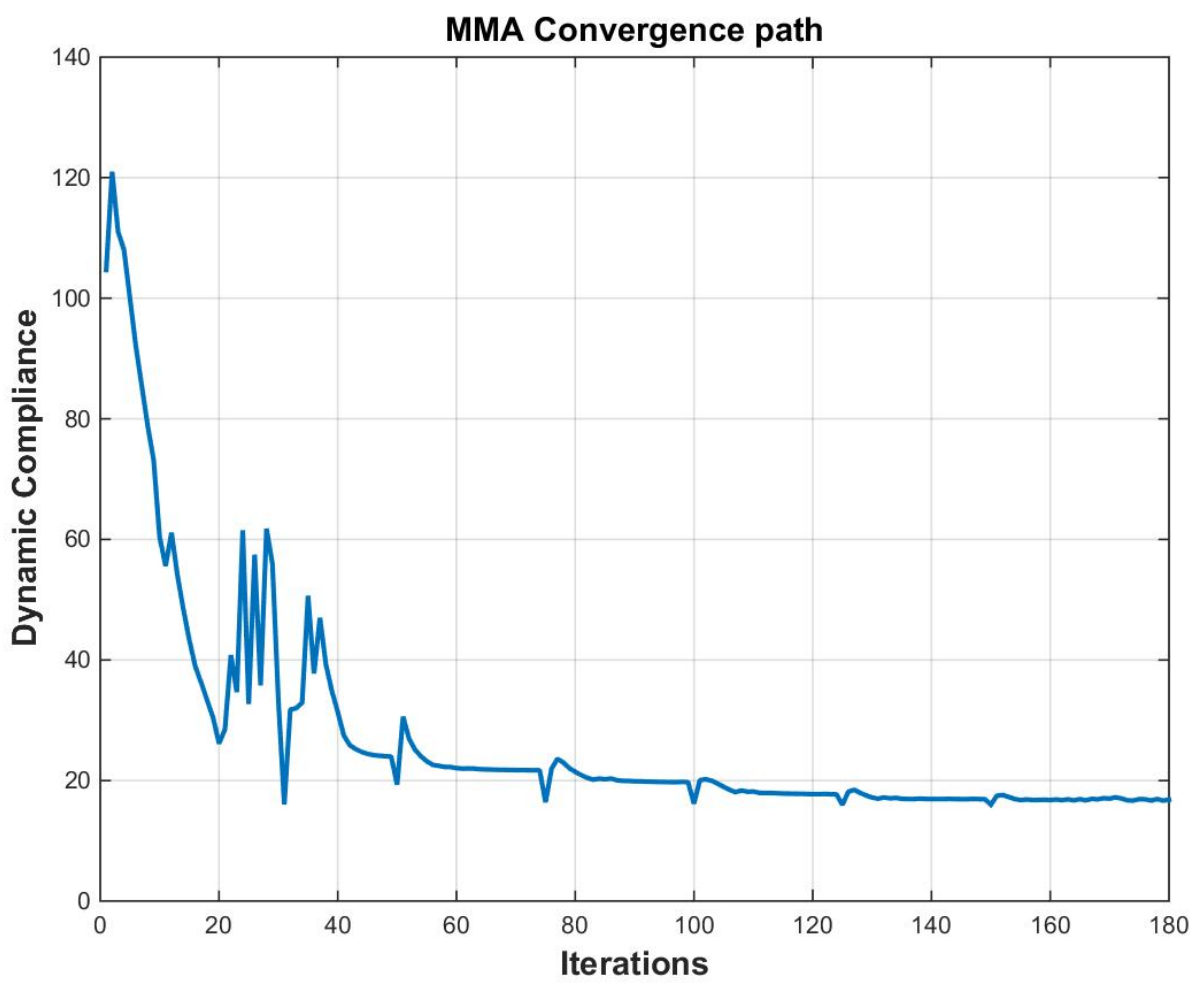

Figure 4: MMA convergence path 
Figure 5 shows the optimal topology for the problem at hand (the right part was drawn by symmetry with respect to the mid vertical axis). Basically no grey regions were experienced and the grey indicator in Equation 34 was found to be 0.0075 and no sharp corners show up as to the solid phase. As to the void phase, no remedy was implemented but the adoption of a multiple phase projection strategy in the spirit of [23] is currently under development.

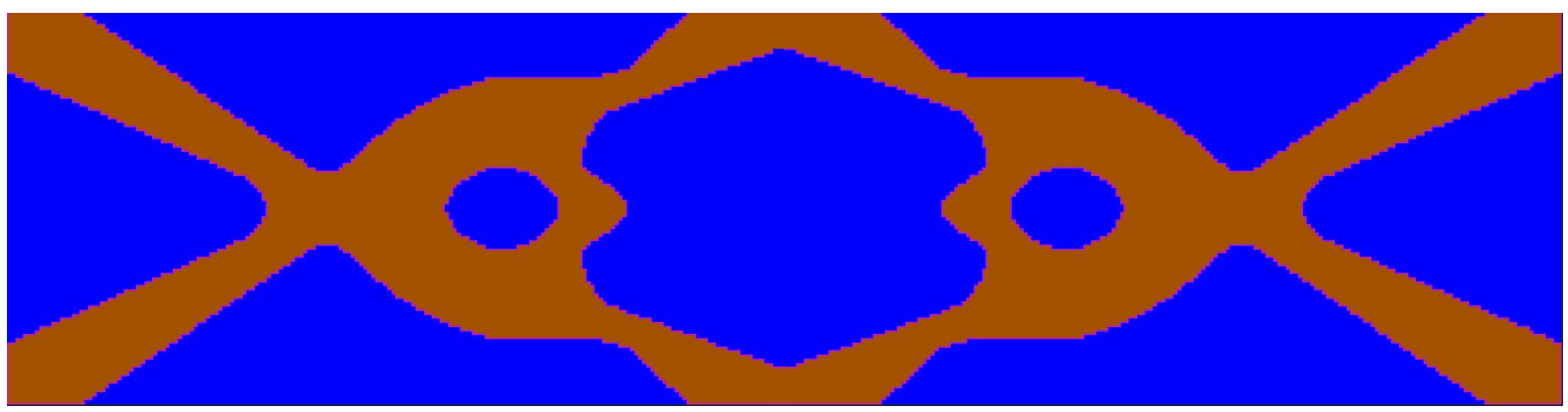

Figure 5: Optimal structure at convergence

Figure 6 shows a comparison between the frequency response functions for the initial uniform structure and the optimal topology at convergence. The peak gain, i.e. $\|\mathscr{C}\|_{\infty}$, is reduced to about one fifth of its initial value as one may also check by looking at Figure 4 that should be considered quite a remarkable performance.

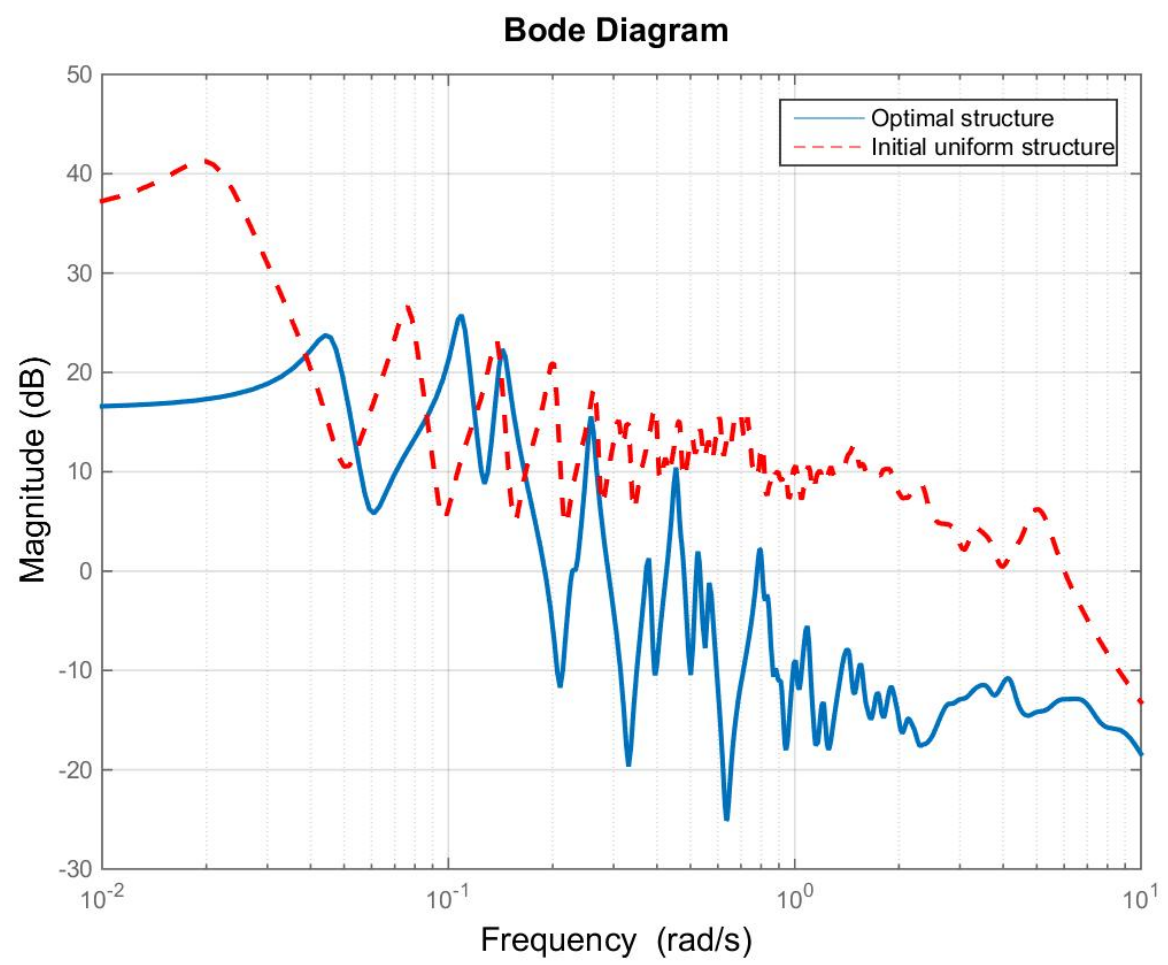

Figure 6: Frequency response functions - Optimal vs initial uniform structure 


\section{CONCLUSIONS}

A new approach to topology optimization of dynamical systems has been introduced that is based on the minimization of the $H_{\infty}$ norm of the input/output transfer function. The framework is abstract enough to include multiple loads as well as multi-output objectives with basically no modifications. Such generality is also enjoyed by the specific dynamical systems object of optimization as long as its governing equations fit a (descriptor) state-space or transfer-function format. For this paper sake a viscoelastic bi-dimensional system in plane strain conditions has been considered obeying a viscoelastic constitutive law and the truly-mixed Arnold-Winther finite element has been used for the spatial discretization. Eventually, the issue of manufacturability of the design has been addressed using the three-field density representation approach $[15,22]$. Extensions to more complex geometries and load conditions are currently under development that include three-dimensional systems and multiple-phase filtering techniques [23].

\section{ACKNOWLEDGMENTS}

Fondazione Cariplo (grant Safer Helmets) is gratefully acknowledged.

\section{REFERENCES}

[1] Z.H.Ma, N. Kikuchi, H.C. Cheng, Topological design for vibrating structures. Computer Methods in Applied Mechanics and Engineering, 121, 259-280, 1995.

[2] J. Du, N. Olhoff, Topological design of freely vibrating continuum structures for maximum values of simple and multiple eigenfrequencies and frequency gaps, Structural and Multidisciplinary Optimization, 34, 91-110, 2007.

[3] N.Olhoff, B. Niu, G. Cheng, Optimum design of band-gap beam structures. International Journal of Solids and Structures, 49, 3158-3169, 2012.

[4] J. Dahl, J. Jensen, O. Sigmund, Topology optimization of transient wave propagation problems in one dimension, Structural and Multidisciplinary Optimization, 36, 585-595, 2008.

[5] J. Jensen, P. Nakshatrala, D. Tortorelli, On the consistency of adjoint sensitivity analysis for structural optimization of linear dynamic problems, Structural and Multidisciplinary Optimization, 49, 831-837, 2014.

[6] M. Pingaro, P. Venini, A fast approach to analysis and optimization of viscoelastic beams, Computers and Structures, 168, 46-55, 2015.

[7] Y. Yu, I.G. Jang and B.M. Kwak, Topology optimization for a frequency response and its application to a violin bridge, Structural and Multidisciplinary Optimization, 48(3), 627-636, 2013.

[8] G.H. Yoon, Structural topology optimization for frequency response problem using model reduction schemes, Computer Methods in Applied Mechanics and Engineering, 199(25), 1744-1763, 2010.

[9] J. Jung, J. Hyun, S. Goo, S. Wang, An efficient design sensitivity analysis using element energies for topology optimization of a frequency response problem, Computer Methods in Applied Mechanics and Engineering, 296, 196-210, 2015. 
[10] M. Bendsoe, O. Sigmund, Topology optimization: Theory, methods and applications. Springer, 2003.

[11] D.P. Giesy, K.B. Lim, $H_{\infty}$ norm sensitivity formula with control system design applications, Journal of Guidance, Control and Dynamics, 16(6), 1138-1145, 1993.

[12] M. Rognes, R. Winther, Mixed finite element methods for linear viscoelasticity using weak simmetry, Mathematical Models and Methods in Applied Sciences, 20(6), 955-985, 2010.

[13] D. Arnold, R. Winther, Mixed finite elements for elasticity, Numerische Mathematik, 92, 401-419, 2002.

[14] P. Venini, Stress constraints and incompressible materials in topology optimization: state of the art and new perspectives, J. Kruis, Y. Tsompanakis and B.H.V. Topping eds. Computational Techniques for Civil and Structural Engineering, Saxe-Coburg Publications, Stirlingshire, UK, Chapter 19, pp 439-465, 2015. doi:10.4203/csets.38.19

[15] B.S. Lazarov, F. Wang, O. Sigmund, Length scale and manufacturability in density-based topology optimization, Archives of Applied Mechanics, 86, 189-218, 2016.

[16] M. Jansen, B.S. Lazarov, M. Schevenels, O. Sigmund, On the similarities between micro/nano lithography and topology optimization projection methods, Structural and Multidisciplinary Optimization, 48, 717-730, 2013.

[17] E. Andreassen, J.S. Jensen, A practical multiscale approach for optimization of structural damping, Structural and Multidisciplinary Optimization, 53, 215-224, 2016.

[18] B.A. Francis, A Course in $H_{\infty}$ Control Theory, Lecture notes in control and information sciences, vol. 88. Springer-Verlag, 1987.

[19] The Mathworks, Inc., Natick, Massachusetts. MATLAB version 8.5.0.197613 (R2015a) 2015.

[20] K. Svanberg, The method of moving asymptotes a new method for structural optimization, International Journal for Numerical Methods in Engineering, 24, 359-373, 1987.

[21] P.Venini, M. Pingaro, A new approach to optimization of viscoelastic beams: minimization of the input/output transfer function $H_{\infty}$-norm, Structural and Multidisciplinary $O p$ timization, under review, 2016.

[22] J.K. Guest, J.H. Prevost, T. Belytschko, Achieving minimum length scale in topology optimization using nodal design variables and projection functions, International Journal for Numerical Methods in Engineering, 61, 238-254, 2004.

[23] J.K. Guest, Topology optimization with multiple phase projection, Computer Methods in Applied Mechanics and Engineering, 199, 123-135, 2009.

[24] E. Andreassen, A. Clausen, M. Schevenels, B.S. Lazarov, O. Sigmund, Efficient topology optimization in MATLAB using 88 lines of code, Structural and Multidisciplinary Optimization, 43, 1-16, 2011. 